\title{
Métodos de avaliação da ciclagem de nutrientes no bioma Cerrado: uma revisão sistemática
}

\author{
Methods of evaluation of nutrient cycling in the Cerrado biome: a systematic \\ review
}

\author{
Jonas Inkotte ${ }^{\mathrm{I}}$, Rosana Carvalho Cristo Martins ${ }^{\mathrm{II}}$, Fernando Paiva Scardua ${ }^{\mathrm{III}}$, \\ Reginaldo Sérgio Pereira ${ }^{\mathrm{II}}$
}

\begin{abstract}
Resumo
Em sistemas florestais, a forma natural de aporte de nutrientes se dá através da serapilheira, a qual compreende todo material vegetal depositado ao solo. Logo, vista a importância do tema, o presente trabalho objetivou realizar uma revisão sistemática sobre as diferentes metodologias utilizadas na temática da serapilheira e ciclagem de nutrientes nas diversas fitofisionomias do bioma Cerrado. Foram avaliadas todas as publicações, independentemente do período de publicação, e catalogadas as que se enquadraram na temática: quantificação da produção e decomposição da serapilheira no bioma Cerrado, por meio de buscas no portal periódicos CAPES. Foram selecionados 26 artigos com estudos nas seguintes formações florestais: Cerrado sentido restrito; Cerradão; Matas de Galeria; Florestas de transição Cerrado-Amazônia e plantios homogêneos. A respeito das metodologias, os coletores mais comuns nos trabalhos de produção foram os de formato quadrado $\left(0,25 \mathrm{~m}^{2}\right)$ e o número de coletores variou bastante entre os trabalhos (de 10 a 60 coletores). As folhas contribuíram em torno de $70 \%$ ou mais do total da serapilheira. A decomposição apresentou grande variação na massa acondicionada nas bolsas e nos tempos de coleta. Com exceção de um trabalho, os manuscritos avaliados não apresentaram estudos sobre a decomposição de outros componentes da serapilheira, que não a foliar, evidenciando assim a necessidade de estudos nesta temática. Os trabalhos apresentaram tempo total de um ano de avaliação em quase sua totalidade, o que evidencia a necessidade dos monitoramentos de longo prazo, pois estudos com breve duração não permitem avaliar os efeitos de alterações interanuais. As metodologias utilizadas nos trabalhos avaliados apresentaram alto grau de variação, evidenciando a necessidade de padronização que permita a comparação entre estes. Por fim, pôde-se observar que algumas fitofisionomias não possuem nenhuma publicação sobre os temas avaliados, o que reforça a necessidade de estudo da dinâmica da ciclagem de nutrientes nestes locais.
\end{abstract}

Palavras-chave: Serapilheira; Decomposição; Aporte; Revisão sistemática

\begin{abstract}
In forest systems, the natural form of nutrient supply is through the litter, which comprises all plant material deposited in the soil. Therefore, the present work aimed to perform a systematic review on the different methodologies used in the litter and nutrient cycling topics in the diverse phytophysiognomies of the Cerrado biome. All publications were evaluated, regardless of the period of publication, and cataloged those that fit the theme: quantification of the production and decomposition of litter in the Cerrado biome, through searches in the Periódicos CAPES gate. Twentysix (26) articles were selected with studies in the following forest formations: Cerrado restricted sense; Cerradão; Gallery Woods; Cerrado-Amazonian transition forests and homogeneous plantations. About the methodologies, the most common collectors in the production works were those with square format $\left(0.25 \mathrm{~m}^{2}\right)$ and the number of collectors varied widely between works (from 10 to 60 collectors). The leaves contributed around $70 \%$ or more of the total litter. The decomposition showed great variation in the mass conditioned in the litter bags and also in times of collection. Except one study, the evaluated manuscripts did not present studies on the decomposition of other litter components, other than the leaves, thus evidencing the need for studies in this topic. The studies presented a total time of one-year evaluation in almost all of them, which shows the need for long-term monitoring, since short-term studies do not allow the evaluation of the inter-annual changes effects. The methodologies used in the evaluated works presented a high degree of variation, evidencing the need for standardization that allows the comparison between them. Finally, it could be observed that some phytophysiognomies do not have any publications on the subjects evaluated, which reinforces the need to study the dynamics of the nutrient cycling in these sites.
\end{abstract}

Keywords: Litter; Decomposition; Litterfall; Systematic quantitative literature review

\footnotetext{
Engenheiro Florestal, MSc., Doutorando do Programa de Pós-Graduação em Ciências Florestais, Faculdade de Tecnologia, Universidade de Brasília, UnB, Campus Universitário Darcy Ribeiro, CEP 70910-900, Brasília (DF), Brasil. jonasink@gmail.com (ORCID: 0000-0001-6151-6658)

II Engenheiro(a) Florestal, Dr (a), Professor(a) do Departamento de Ciências Florestais, Universidade de Brasília, Faculdade de Tecnologia, Campus Universitário Darcy Ribeiro, CEP 70910-900, Brasília (DF), Brasil. rccristo@unb.br (ORCID: 0000-0001-8066-7566) / reginaldosp@unb.br(ORCID: 0000-0002-6614-6825)

III Engenheiro Florestal, Dr., Professor da Universidade de Brasília, Faculdade Gama, Área Especial de Indústria Projeção A - Setor Leste, CEP 72.444-240, Gama (DF), Brasil. fscardua@unb.br (ORCID: 0000-0003-4949-3283)
} 


\section{Introdução}

O entendimento do funcionamento e variáveis que influenciam a estabilidade de uma comunidade vegetal é crucial para que se possa manejá-las buscando a sustentabilidade dos ecossistemas florestais. E, dentre estas, a produção e decomposição da serapilheira são fundamentais para funcionalidade ecossistêmica e o estabelecimento destes ecossistemas. Parte do processo de retorno da matéria orgânica e dos nutrientes ao solo ocorre pela produção de serapilheira, sendo este o meio natural mais importante da transferência de elementos necessários ao crescimento e desenvolvimento das plantas (LOPES et al., 2009).

A serapilheira é um dos componentes mais importantes de um ecossistema florestal, contemplando todo material vegetal depositado ao solo pelas árvores, como: folhas, galhos, e estruturas reprodutivas (flores, sementes, frutos), além de outros materiais orgânicos de origem não vegetal. Estes materiais produzidos e posteriormente depositados no solo florestal proporcionam a ciclagem de nutrientes, que por meio de sua decomposição liberam os nutrientes absorvidos pelas plantas (COSTA et al., 2010).

Este processo de ciclagem de nutrientes é de suma importância em solos com alto intemperismo e baixos teores nutricionais, como é o caso de muitos solos brasileiros, nos quais a biomassa vegetal é o principal reservatório de nutrientes. Dentre os diversos tipos de solos ocorrentes no Brasil, destacam-se os Latossolos, os quais compreendem entre 32 e $46 \%$ do território nacional, sendo solos muito intemperizados e com altas taxas de lixiviação, estes ocorrem em locais planos e com relevo suave, sendo geralmente solos ácidos, bem profundos e pobres na disponibilidade de nutrientes devido aos intensos processos de intemperismo (FONTES, 2012).

Os solos predominantes dos Cerrados se enquadram nestas características e originalmente apresentam deficiências nutricionais e são classficados como Latossolos distróficos com alta saturação de Al. Esta baxa fertilidade reflete na menor concentração de nutrientes nas folhas das espécies nativas ocorrentes nestes solos, o que reforça a necessidade de estudos sobre a ciclagem de nutrientes nas diferentes fitofisionomias deste bioma (HARIDASAN, 2000).

Existem diferentes tipos de estudos de revisão de literatura, e dentre estes, está a revisão sistemática ou "systematic quantitative assessment", proposta por Pickering e Byrne (2014), a qual utiliza uma padronização na busca e catálago de todos os artigos encontrados, para a posterior análise quantitativa, evitando assim, erros de amostragem por utilizar um padrão de pesquisa. Este tipo de revisão proporciona ao pesquisador mapear os limites eixistentes na literatura dentro de cada tema escolhido, permitindo indentificar onde as generalizações ocorrem (locais em que a maior parte dos estudos vem sendo realizados) e suas limitações - lacunas não preenchidas dentro de determinados temas de estudo - o que facilita o avanço das pesquisas, além de poder verificar as possíveis discrepâncias metodológicas dentro de uma mesma linha de pesquisa (BORENSTEIN et al., 2009).

Sendo assim, o presente estudo teve como objetivo caracterizar os trabalhos de produção e decomposição da serapilheira no bioma Cerrado quanto a sua forma de publicação e as metodologias utilizadas e seus resultados, buscando compreender sua área de abrangência em cada fitofisionomia para verificar quais assuntos possuem potencial para novas linhas de pesquisa.

\section{Desenvolvimento}

\section{Descrição dos procedimentos metodológicos}

Os procedimentos metodológicos que conduziram o presente trabalho fundamentamse nos estudos de revisão sistemática propostos por Pickering e Byrne (2014). O levantamento 
utilizou como fonte de dados todos os artigos publicados em revistas científicas que apresentavam correlação com a temática acerca da serapilheira, buscando identificar sua presença e relação como o foco de estudo. A busca ocorreu na base de dados Periódicos CAPES, por ser uma das maiores bases de dados, contendo artigos nacionais e internacionais.

O levantamento dos trabalhos ocorreu no período entre março e junho de 2017, utilizando o mecanismo de "buscar assunto" do banco de dados dos Periódicos CAPES, e dentro deste, a escolha da ferramenta intitulada "Busca avançada". Os termos inseridos como palavras-chave foram: "Serapilheira e Cerrado"; "Litterfall and Cerrado", sendo então analisados todos os trabalhos científicos que foram encontrados, sem definição de um período de tempo, compreendendo assim, todas as publicações catalogadas na plataforma até a data da realização do levantamento. Cabe ressaltar que as pesquisas foram realizadas nos domínios da Universidade de Brasília - UnB com acesso total e irrestrito ao banco de dados da Periódicos CAPES.

O critério de inclusão dos artigos encontrados nas buscas se deu pelo enquadramento ou não nos temas específicos preestabelecidos, compreendendo assim todos os trabalhos referentes à quantificação da produção e decomposição da serapilheira nas áreas dentro do bioma Cerrado, sendo elas compostas por áreas de formação florestal nativa ou plantada.

Após tal triagem, os aspectos gerais dos artigos incluídos na base dados foram analisados sob os seguintes aspectos: ano de publicação dos artigos, o meio de publicação, ou seja, quais revistas científicas publicaram os artigos; os locais em que foram realizados os estudos, com a finalidade de conhecer quais fitofisionomias foram mais avaliadas. Por fim, os artigos foram divididos em duas áreas específicas dentro da temática da serapilheira: os trabalhos que abordavam a deposição/aporte/produção de serapilheira e os estudos envolvendo as taxas de decomposição de serapilheira.

Dentro de cada área específica, foram avaliadas as metodologias utilizadas, sendo assim necessária a abertura de subdivisões para melhor perceber suas semelhanças ou diferenças. Os trabalhos referentes ao aporte de serapilheira foram avaliados em relação ao formato e área do coletor, quanto à forma da classificação dos materiais vegetais na triagem e a quantificação da contribuição destas diferentes estruturas. Os artigos sobre a decomposição da serapilheira foram avaliados quanto à quantidade e o tipo de material vegetal adicionado nas bolsas de decomposição (litter bags), tempos de exposição da primeira coleta e tempo total de exposição dos litter bags.

Cabe ressaltar que os trabalhos realizados em ambientes aquáticos não fizeram parte das análises por possuírem implicações, metodologias e formas de análise diferentes dos aplicados nos ambientes terrestres.

\section{Resultados das buscas e enquadramento dos artigos nos critérios de inclusão}

Os estudos sobre a quantificação da produção e as taxas de decomposição foram catalogados e analisados, sendo encontrados 77 artigos científicos, para a busca com os termos "Serapilheira and Cerrado" e que dentre os quais, 11 se enquadraram nas categorias preestabelecidas - nove destes em áreas de Cerrado nativo e dois em florestas plantadas em áreas orginalmente ocupadas por Cerrado. Utilizando os termos "Cerrado and Litterfall" foram encontrados 252 resultados, sendo que destes, 15 se enquadraram nas categorias, das quais 12 destes foram realizados em áreas de Cerrado nativo, três em florestas plantadas em áreas orginalmente ocupadas por Cerrado e um em áreas de plantio de pinus comparado a áreas de Cerrado nativo. Os demais artigos foram descartados em virtude de não se enquadrarem nos critérios preestabelecidos referentes à temática analisada, resultando em um total de 26 artigos catalogados e analisados.

Aspectos gerais dos manuscritos avaliados

Os trabalhos realizados sobre a ciclagem de nutrientes que se enquadraram nos parâmetros 
de seleção anteriormente descritos, entre florestas plantadas em áreas originais de Cerrado em diferentes fitofisionomias do bioma em seu estado natural totalizaram 26 artigos publicados em 16 diferentes revistas, sendo que quatro delas foram responsáveis por quase a metade do total das publicações (46,15\%), sendo elas: Forest Ecology and Management, Brazilian Journal of Biology, Plant and Soil e Plant Ecology com três publicações cada. O fato de tais revistas possuírem alto fator de impacto e serem revistas internacionais ressalta a importância da temática do estudo.

Avaliando as datas de publicações dos trabalhos pôde-se perceber notório aumento no número de trabalhos nos últimos seis anos, o que evidência uma tendência de crescimento nos trabalhos sobre o referido tema (Figura 1). Destacam-se os anos de 2011, 2012 e 2016 com sete, três e três publicações, respectivamente.

\section{Figura 1 - Evolução do número de publicações analisadas com o passar dos anos.}

Figure 1 - Evolution in the number of publications analyzed over the years.

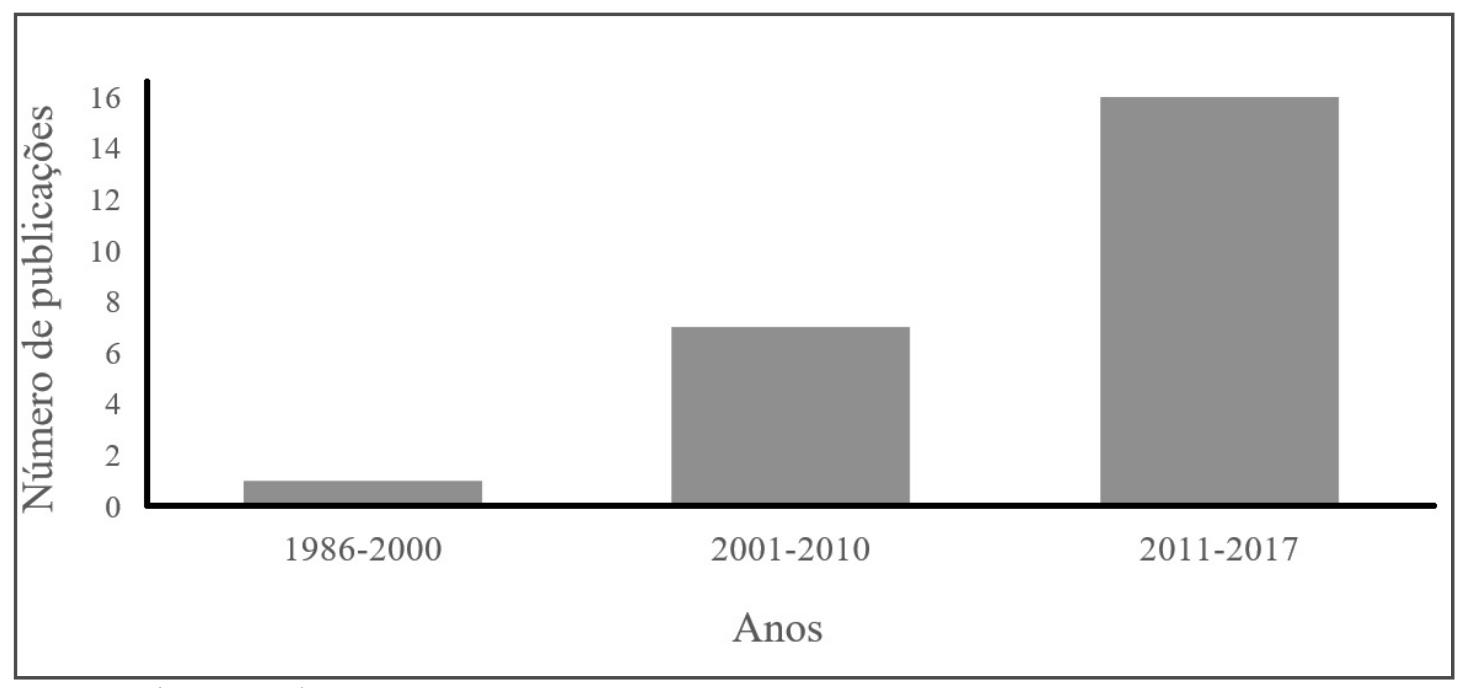

Fonte: Inkotte et al. (2017)

Dos 26 manuscritos avaliados, a temática do aporte/deposição/produção da serapilheira foi abordada em 22 trabalhos, perfazendo ampla maioria. Já a quantificação das taxas de decomposição foi abordada em 13 publicações. Cabe ressaltar que muitos dos artigos apresentavam análise de ambas temáticas de forma simultânea, o que beneficia a consistência dos trabalhos a respeito da dinâmica da ciclagem de nutrientes.

O bioma Cerrado é composto por diversas fitofisionomias e classificada de formas diferentes dependendo de cada autor, conforme relatado por Bastos e Ferreira (2010). Escolheuse então utilizar uma adaptação da classificação proposta por Ribeiro e Walter (2008), a qual utiliza um total de 11 diferentes fitofisionomias, como base, sendo assim classificadas as áreas de trabalho dos manuscritos (Figura 2), entretanto, destas, foram registrados trabalhos em apenas quatro delas, o que evidencia uma lacuna para novos estudos nas demais fitofisionomias ainda não avaliadas até o momento. Foram realizados 12 estudos em áreas de Cerrado sentido restrito; cinco em áreas de Cerradão; quatro em Matas de galeria e cinco em Florestas de transição Cerrado Amazônia. Com relação aos estudos desenvolvidos em plantios homogêneos de espécies exóticas, em área natural de Cerrado, foram cinco trabalhos em áreas de plantio de Eucalyptus sp. e dois trabalhos em plantios de outras espécies, uma área de plantio de Pinus caribaea e uma área de plantio de Acacia mangium. 


\section{Figura 2 - Análise das fitofisionomias e usos de solo avaliados nos manuscritos.}

Figure 2 - Analysis of the phytophysiognomies and soil uses evaluated in the manuscripts.

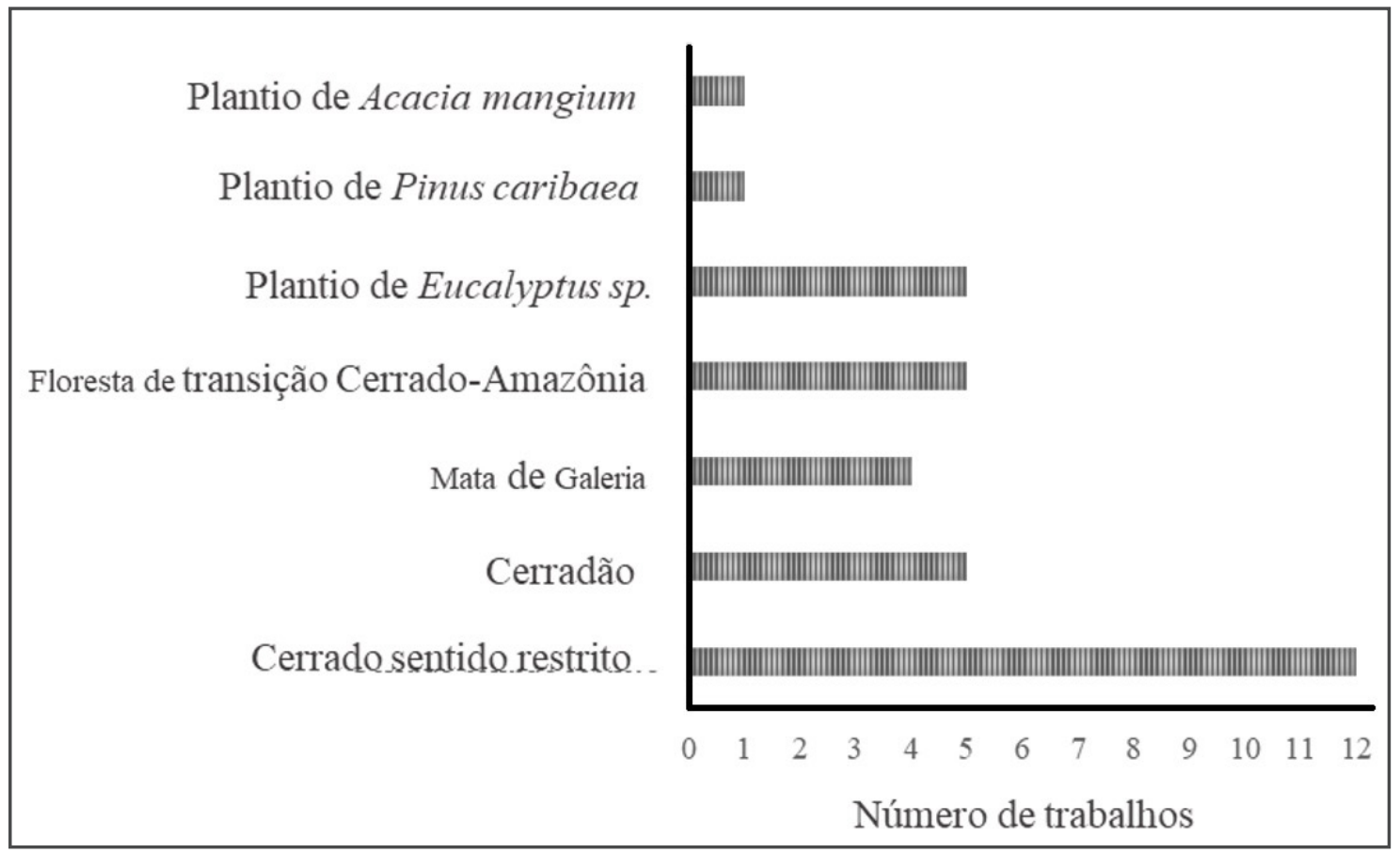

Em que: $\mathrm{N}$ = Número de trabalhos.

Fonte: Inkotte et al. (2017)

\section{Análise dos trabalhos de aporte da serapilheira no Cerrado}

Os 22 manuscritos referentes ao aporte de serapilheira no bioma Cerrado foram avaliados com enfoque nas metodologias utilizadas, suas composições florestais e resultados. Dentre os trabalhos avaliados, foram realizados experimentos em nove áreas de Cerrado sentido restrito, quatro em áreas de Mata de galeria, quatro em áreas de Cerradão, quatro em Florestas de transição entre os biomas Cerrado e Amazônia, cinco em áreas de plantios de Eucalipto, dois artigos de revisão (sem experimentos a campo), e um em área de Pinus caribaea e uma área de plantio de Acacia mangium.

Fica evidente que a formação florestal com o maior número de trabalhos sobre a quantificação da produção de serapilheira foram as áreas de Cerrado Sentido restrito, indicando que as demais formações necessitam de mais estudos, gerando assim, resultados mais confiáveis e passíveis de comparação entre as mesmas formações e outras fitofisionomias do bioma Cerrado. Tal fato auxiliará em um maior entendimento dos processos que contribuem para a sustentabilidade e diversidade entre os ecossistemas. Cabe ressaltar que algumas fitofisionomias não possuem nenhum tipo de trabalho com essa temática, o que ressalta a necessidade da expansão destes estudos dentro do bioma Cerrado entre seus diferentes ecossistemas.

O tempo de coleta de dados variou bastante entre os trabalhos conforme pode ser observado na Tabela 1, entretanto, uma grande maioria realizou as coletas com o tempo total de experimento de um ano, totalizando 10 trabalhos. Com dois anos de avaliação, cinco trabalhos foram publicados, outros três manuscritos analisaram a decomposição por três anos e apenas um trabalho ultrapassou o período de três anos, em um estudo com 8 anos realizado por Laclau et al. (2010), em povoamentos de Acacia mangium e Eucalyptus sp. A incidência reduzida de trabalhos de médio e longo prazo evidenciam 
a necessidade da realização de avaliações mais duradouras, uma vez que muitas espécies possuem produções de flores, frutos e folhas sazonais e com alterações interanuais (RUBIM; NASCIMENTO; MORELLATO, 2010; ZIMMERMAN et al., 2007).

\section{Tabela 1 - Classificação dos manuscritos avaliados quanto ao tempo total de exposição das bolsas de decomposição.}

Table 1 - Classification of the evaluated papers regarding the total time of exposure of the decomposition bags.

\begin{tabular}{lcc}
\hline Tempo de estudo & $\mathbf{N}$ & Referências \\
\hline $\mathbf{1}$ Ano & 10 & $\begin{array}{c}\text { Aquino et al. (2016); Paiva; Silva; Haridasan (2015); Epron et al. (2012); } \\
\text { Butler et al. (2012); Epron et al. (2011); Silva et al. (2009); Quesada et al. } \\
(2008) ; \text { Kozovits et al. (2007); Silva et al. (2007); Cianciaruso et al. (2006) }\end{array}$ \\
\hline $\mathbf{2}$ anos & 5 & $\begin{array}{c}\text { Oliveira et al. (2017); Villalobos-Veja et al. (2011); Balch et al. (2008); } \\
\text { Valenti; Cianciaruso; Batalha (2008); Wilcke e Lilienfein (2002); }\end{array}$ \\
\hline $\mathbf{3}$ anos & 3 & Silva; Poggiani e Laclau (2011); Almeida et al. (2010); Sanches et al. (2009) \\
\hline $\mathbf{8}$ anos & 1 & Laclau et al. (2010) \\
\hline
\end{tabular}

Em que: $\mathrm{N}=$ Número de trabalhos.

A utilização de coletores é a metodologia mais comumente adotada nos trabalhos referentes à produção de serapilheira, pois permite a quantificação do material aportado em uma pequena área, a qual será posteriormente extrapolada para hectare. Normalmente, os resultados são apresentados nas unidades de medida: Mg (Megagrama) hectare ${ }^{-1}$ ano $^{-1}$ ou ton hectare ${ }^{-1}$ ano $^{-1}$ (SCORIZA et al., 2012). Alguns aspectos importantes devem ser levados em consideração, para a padronização dos procedimentos metodológicos, como é o caso do número de coletores instalados por uma determinada área, formato dos coletores - mesmo que estas diferenças sejam diluídas na extrapolação para área de um hectare - posicionamento dos coletores na área de estudo, entre outros.

Com relação ao número de coletores instalados em cada experimento, os trabalhos divergiram bastante entre si, tendo trabalhos como apontado em Silva et al. (2007), com 10 coletores por tratamento e trabalhos com 60 e 52 coletores por tratamento, conforme Aquino et al. (2016) e Paiva, Silva e Haridasan (2015), respectivamente. Ficando assim evidenciado que não há um consenso a respeito do número de coletores a serem utilizados, o que também pode gerar alterações na amostragem da serapilheira aportada.

Os resultados das análises referentes ao formato dos coletores e suas dimensões podem ser visualizados na Tabela 2. Puderam-se verificar basicamente dois tipos de metodologias distintas: os coletores com formato circular e os coletores com formato quadrado. O formato mais utilizado foi o quadrado, com um total de 15 trabalhos que optaram por este tipo de coletor (cerca de $68,2 \%$ dos trabalhos), outras três publicações utilizaram coletores de formatos circulares/ cônicos (13,65\%) e outros quatro não descreveram o formato utilizado.

As dimensões dos coletores encontradas nos trabalhos avaliados variaram bastante, indo de $0,02 \mathrm{~m}^{2}$ até $1,00 \mathrm{~m}^{2}$. Ainda, mesmo dentro do mesmo tipo de coletor (formato), as dimensões utilizadas apresentam muitas diferenças, cujos coletores quadrados variaram entre $0,21 \mathrm{~m}^{2}$ e 1,00 $\mathrm{m}^{2}$, e essa diferença foi ainda maior para os coletores circulares, os quais variaram entre $0,02 \mathrm{e}$ $0,44 \mathrm{~m}^{2}$.

A escolha das dimensões e formatos dos coletores de aporte de serapilheira, de acordo com Scoriza et al. (2012) fica a cargo de cada pesquisador, uma vez que a quantificação do material 
aportado é feita por meio de equação na qual a área do coletor é uma variável. Entretanto, os diferentes formatos e dimensões podem acabar implicando em alterações na amostragem da serapilheira aportada, facilitando ou dificultando a queda dos materiais vegetais nos coletores.

Tabela 2 - Caracterização dos coletores utilizados nos trabalhos de aporte da serapilheira.

Table 2 - Characterization of the collectors used in the litterfall works.

\begin{tabular}{|c|c|c|c|}
\hline Formato & Dimensões & $\mathbf{N}$ & Referências \\
\hline \multirow{4}{*}{ Quadrado } & $0,25 \mathrm{~m}^{2}$ & 7 & $\begin{array}{l}\text { Paiva; Silva; Haridasan (2015); Silva et al. (2011); Villalobos- } \\
\text { Veja et al. (2011); Valenti, Cianciaruso e Batalha (2008); } \\
\text { Kozovits et al. (2007); Cianciaruso et al. (2006); Wilcke; } \\
\text { Lilienfein (2002) }\end{array}$ \\
\hline & $0,21 \mathrm{~m}^{2}$ & 2 & Almeida et al. (2010); Laclau et al. (2010) \\
\hline & $0,50 \mathrm{~m}^{2}$ & 3 & Epron et al. (2012); Epron et al. (2011); Balch et al. (2008) \\
\hline & $1,00 \mathrm{~m}^{2}$ & 3 & Sanches et al. (2009); Silva et al. (2009); Silva et al. (2007) \\
\hline \multirow{4}{*}{$\begin{array}{l}\text { Circular } \\
\text { Funil }\end{array}$} & $0,28 \mathrm{~m}^{2}$ & 1 & Oliveira et al. (2017) \\
\hline & $0,44 \mathrm{~m}^{2}$ & 1 & Butler et al. (2012) \\
\hline & $0,02 \mathrm{~m}^{2}$ & 1 & Quesada et al. (2008) \\
\hline & $0,33 \mathrm{~m}^{2}$ & 1 & Aquino et al. (2016) \\
\hline $\begin{array}{l}\text { Não } \\
\text { especificado }\end{array}$ & Não especificado & 3 & $\begin{array}{c}\text { Bustamante et al. (2012); Parron; Bustamante; Arkewitz (2011); } \\
\text { Lathwell; Grove (1986) }\end{array}$ \\
\hline
\end{tabular}

Em que: N.E. = Não especificado.

Tais discrepâncias metodológicas podem dificultar a comparação entre diferentes trabalhos com fidedignidade, pois, mesmo que ainda as áreas dos coletores sirvam como uma amostra para posterior extrapolação para área de um hectare, as florestas, mas em específico o Cerrado, apresentam formações fisionômicas bastante distintas entre si, e isto implica em uma maior variabilidade de produção intra e interespecífica, devido aos diferentes padrões nas composições florísticas encontradas.

Ainda, mesmo dentro de um mesmo local, a variação espacial pode se tornar um fator importante, o que foi constatado por Aquino et al. (2016) em um experimento realizado em uma Mata de galeria do córrego Lava-pés, em Ipameri - GO, onde os autores verificaram a existência de dependência espacial na produção de serapilheira, para o total depositado e para a fração folhas, o que também não foi levado em conta na grande maioria das publicações desta temática.

A produção anual de serapilheira foi avaliada comparando estudos realizados dentro da mesma fitofisionomia do bioma Cerrado, a qual apresentou valores bastante distintos entre os trabalhos. Para os estudos realizados em áreas de Cerrado sentido restrito, os valores variaram entre 0,62 Mg hectare ${ }^{-1}$ ano $^{-1}$ encontrado em Silva et al. (2007) e 5,8 Mg hectare ${ }^{-1}$ ano $^{-1}$ conforme trabalho de Valenti, Cianciaruso e Batalha (2008). Para as áreas de Cerradão a variação foi ainda maior, indo de $1,04 \mathrm{Mg}_{\text {hectare }}^{-1}$ ano $^{-1}$, conforme apresentado por Silva et al. (2007) e 9,27 Mg hectare ${ }^{-1}$ ano $^{-1}$ conforme descrito em Oliveira et al. (2017). Estas variações bastante amplas podem estar atreladas a diversos fatores, como: estado de conservação e estágio sucessional da floresta estudada, posição espacial da alocação dos coletores, condições microclimáticas diferentes, ocorrência de diferentes composições florísticas dentro da mesma fitofisionomia, com espécies com maior ou menor produção de serapilheira, entre outros fatores (GIÁCOMO; PEREIRA; MACHADO, 2012; NASCIMENTO; CERQUEIRA; HENDERSON, 2015; AQUINO et al., 2016).

Alguns trabalhos de produção da serapilheira apresentaram além da quantificação do 
aporte total anual, a triagem dos materiais vegetais em diferentes frações, de acordo com sua composição anatômica, e têm por finalidade a verificação da contribuição de cada fração da serapilheira no aporte total. Nos trabalhos analisados, 16 estudos apresentaram algum tipo de classificação (aproximadamente 72,7\%), entretanto, os trabalhos utilizaram classificações bastante distintas entre si, e muitos deles não quantificaram a contribuição das diferentes frações no total depositado, conforme apresentado na Tabela 3.

\section{Tabela 3 - Tipos de classificação dos materiais vegetais aportados observados nos manuscritos avaliados.}

Table 3 - Types of classification of the litterfall observed in the evaluated papers.

\begin{tabular}{lcc}
\hline \multicolumn{1}{c}{$\begin{array}{c}\text { Triagem do material } \\
\text { aportado }\end{array}$} & N & Referências \\
\hline $\begin{array}{l}\text { Folhas, Ramos, Estruturas } \\
\text { reprodutivas e Miscelânea }\end{array}$ & 7 & $\begin{array}{c}\text { Oliveira et al. (2017); Aquino et al. (2016); Sanches et al. (2009); } \\
\text { Silva et al. (2009); Valenti; Cianciaruso; Batalha (2008); Silva et } \\
\text { al. (2007); Cianciaruso et al. (2006) }\end{array}$ \\
\hline Só Folha & 4 & $\begin{array}{c}\text { Butler et al. (2012); Silva; Poggiani; Laclau (2011); Almeida et al. } \\
\text { (2010); Kozovits et al. (2007) }\end{array}$ \\
\hline $\begin{array}{l}\text { Folhas, Cascas e Galhos } \\
\text { mortos }\end{array}$ & 2 & Epron e al. (2012) e Epron et al. (2011) \\
\hline $\begin{array}{l}\text { Folhas e Miscelânea (frutos, } \\
\text { galhos e etc.) }\end{array}$ & 1 & Paiva; Silva; Haridasan (2015) \\
\hline Retirada de Galhos $>\mathbf{1} \mathrm{cm}$ & 1 & Balch et al. (2008) \\
\hline $\begin{array}{l}\text { Folhas, Estruturas } \\
\text { reprodutivas e Outros } \\
\text { materiais }\end{array}$ & 1 & Villalobos-Vega et al. (2011) \\
\hline \multicolumn{1}{c}{ Total } & 16 & \\
\hline
\end{tabular}

Em que: $\mathrm{N}$ = Número de trabalhos.

A classificação dos materiais vegetais aportados nas frações "folhas, ramos, estruturas reprodutivas e miscelânea" foi a forma de classificação mais comum, sendo evidenciada quase metade do total dos trabalhos avaliados $(43,75 \%)$, seguidos pela quantificação da contribuição somente foliar com um quarto dos trabalhos avaliados. Levando em consideração que tais diferenças metodológicas já vêm ocorrendo há um certo tempo, e não só nos trabalhos referentes ao bioma Cerrado, mas em todas as formações florestais, Scoriza et al. (2012) recomendam o preestabelecimento da forma de classificação destes materiais, com base em uma revisão científica, possibilitando desta forma, realizar comparações com outros trabalhos de forma mais apropriada.

Os manuscritos que realizaram a triagem do material vegetal aportado, algumas vezes, realizaram também a quantificação da participação de cada uma destas classes de material vegetal aportado, no entanto, esta prática foi realizada em poucos trabalhos. Os valores encontrados para a contribuição das folhas no total produzido variaram entre 42,92 e 75,3\% nas áreas de Cerrado sensu stricto, sendo que os valores em torno de $75 \%$ foram mais comuns. O valor de $42,92 \%$ apresentado no trabalho de Silva et al. (2007) encontra-se bastante abaixo dos demais resultados apresentados nas outras áreas de Cerrado sentido restrito, tal fato está possivelmente atrelado à alta contribuição de frutos no total produzido, com $32,31 \%\left(201 \mathrm{~kg} \mathrm{ha}^{-1} \mathrm{ano}^{-1}\right)$. Já a 
quantificação da participação dos galhos na serapilheira ficou abaixo de $20 \%$, aproximadamente entre 17,51 e $18 \%$.

Com relação à contribuição da fração de folhas, independentemente da fitofisionomia, os resultados apresentaram valores semelhantes, todos com contribuições acima dos $70 \%$. Para os três trabalhos realizados em áreas de Cerradão, os resultados variaram entre 72,27 e 82,95\%. Os trabalhos em Matas de Galeria apresentaram valores entre 71,50\% e 75\%. Já nas florestas de transição entre Amazônia e Cerrado, os valores da contribuição das folhas na composição da serapilheira foram de $70 \%, 84,48 \%$ e um trabalho apontando uma contribuição da fração folha com valores entre 60 a $93 \%$ da produção total. Estes valores superiores nas áreas de cerradão, floresta de transição e mata de galeria em comparação ao Cerrado sentido restrito se dá devido à diferente composição florística e dossel mais fechado (RIBEIRO; WALTER, 2008), o que consequentemente acarreta em uma maior produção de serapilheira.

A maior parte dos valores encontrados nos trabal hos realizados nos diferentes ecossistemas do bioma Cerrado condizem com o que afirma Poggiani (2012), que por meio de estudos em diversos biomas florestais apontaram que a média da contribuição das folhas na serapilheira varia entre 60 a $80 \%$ do total da produção. Cabe ressaltar que a análise dos demais componentes da serapilheira não foram avaliados em razão da grande diferença em sua classificação, ficando assim evidenciado a necessidade de uma uniformidade entre os estudos, mesmo que de forma mais abrangente como para todo um bioma, possibilitando assim a comparação entre os trabalhos.

\section{Análise dos trabalhos de decomposição da serapilheira no Cerrado}

Referente aos trabalhos sobre a decomposição da serapilheira nas áreas do bioma Cerrado, sete em Cerrado sentido restrito foram avaliadas, quatro em áreas de Cerradão, três experimentos em Floresta de transição entre Cerrado e Amazônia e outros dois em áreas de plantios homogêneos de Eucalipto. Cabe ressaltar que não existe nenhum tipo de trabalho a respeito da quantificação da decomposição nas demais fitofisionomias do bioma Cerrado, o que ressalta a necessidade da expansão destes estudos dentro do bioma Cerrado entre seus diferentes ecossistemas.

Para a realização deste tipo de trabalho, a utilização de bolsas de decomposição ou "litter bags" é a prática mais comum, onde uma porção de material vegetal (geralmente folhas) é acondicionada nestas bolsas, e a partir da diferença da massa inicial e a massa final após algum tempo de exposição destas bolsas a campo é realizada a quantificação da decomposição da serapilheira. Após esta quantificação, por meio de uma equação é então obtida a taxa de decomposição (k), normalmente anual (WIEDER; LANG, 1982).

Ao analisar os trabalhos puderam-se constatar quatro dimensões distintas na confecção das bolsas de decomposição, variando entre $5 \times 5 \mathrm{~cm}$ e $30 \times 30 \mathrm{~cm}$, sendo o tamanho mais comum o de 20 x $20 \mathrm{~cm}$ (cinco dos 13 trabalhos avaliados). Além das dimensões das "litter bags", as malhas de nylon utilizadas na confecção divergiram entre o tamanho de suas aberturas, onde as mais comuns foram as de $2 \mathrm{~mm}$ e $1 \mathrm{~mm}$, conforme apresentado na Tabela 4 . A abertura das malhas tem interferência direta na decomposição, uma vez que suas dimensões determinará a entrada ou não da fauna edáfica decompositora (SETALA; MARSHALL; TROFYMOW, 1996).

Sendo assim, recomenda-se utilizar aberturas maiores como as de $2 \mathrm{~mm}$, e evitar espaços muito maiores que este, pois ao deslocar as bolsas para o campo, pode-se perder material acondicionado nas bolsas e ocasionar erros nas determinações da decomposição.

Apenas um dos trabalhos não utilizou a metodologia de "litter bags", que foi o de Miatto e Batalha (2016), no qual os autores utilizaram a metodologia de tea bags, proposto por Keuskamp et al. (2013), em que sacos de folhas são enterrados no solo e retirados após três meses de decomposição destas.

A quantidade de gramas de material vegetal acondicionados nas bolsas de decomposição foi avaliada nos trabalhos analisados nesta revisão sistemática, com seus resultados apresentados na Tabela 5. A utilização de cinco g por bolsa foi a metodologia mais comum entre os trabalhos, 
sendo observada em cinco publicações, seguida por quatro trabalhos que utilizaram $10 \mathrm{~g}$. Outro trabalho utilizou $20 \mathrm{~g}$ de material vegetal por bolsa e, contrariando o meio comum, dois trabalhos apresentaram intervalos de valores para a quantidade de gramas por litter bag, com valores entre 2-5 g e 3-5 g. Tais alternâncias nas massas das bolsas podem acarretar em grandes variações nos dados coletados, em caso de não se ter um controle rigoroso da quantificação do peso destas bolsas, uma vez que a velocidade de decomposição e sua taxa k serão estimadas por meio destes valores.

Tabela 4 - Quantificação dos trabalhos de acordo com as dimensões e abertura das malhas de nylon das bolsas de decomposição.

Table 4 - Quantification of the works according to the dimensions and opening of the nylon mesh of the litter bags.

\begin{tabular}{|c|c|c|}
\hline Dimensões & $\begin{array}{c}\text { Abertura da } \\
\text { malha }\end{array}$ & Referências \\
\hline N.E. & $1 \mathrm{~mm}$ & Carvalho et al. (2014) \\
\hline N.E. & N.E. & Freitas; Cianciaruso; Batalha (2012) \\
\hline N.E. & N.E. & Bambi et al. (2011) \\
\hline N.E. & $1 \mathrm{~mm}$ & Valenti; Cianciaruso; Batalha (2008) \\
\hline $20 \times 20 \mathrm{~cm}$ & $1 \mathrm{~mm}$ & Cianciaruso et al. (2006) \\
\hline $20 \times 20 \mathrm{~cm}$ & $1 \mathrm{~mm}$ & Villalobos-Vega et al. (2011) \\
\hline $20 \times 20 \mathrm{~cm}$ & $2 \mathrm{~mm}$ & Kozovits et al. (2007) \\
\hline $20 \times 20 \mathrm{~cm}$ & $5 \mathrm{~mm}$ & Silva; Poggiani; Laclau (2011) \\
\hline $20 \times 20 \mathrm{~cm}$ & $2 \mathrm{~mm}$ & Laclau et al. (2010) \\
\hline $20 \times 24 \mathrm{~cm}$ & $2 \mathrm{~mm}$ & Oliveira et al. (2017) \\
\hline $30 \times 30 \mathrm{~cm}$ & N.E. & Sanches et al. (2009) \\
\hline $30 \times 30 \mathrm{~cm}$ & $2 \mathrm{~mm}$ & Silva et al. (2009) \\
\hline $5 \times 5 \mathrm{~cm}$ (tea bag) & $0-25 \mathrm{~mm}$ & Miatto; Batalha (2016); \\
\hline
\end{tabular}

Em que: $\mathrm{N}=$ Número de trabalhos.

Com a constatação de tão grande variação nas massas iniciais das bolsas, pôdese evidenciar uma falta de padrão na quantidade de material acondicionado nas bolsas de decomposição, o que pode de alguma forma influenciar nas taxas $\mathrm{k}$ estimadas, bem como na ação dos organismos decompositores, sendo assim, necessária uma padronização para evitar possíveis desvios e informações desencontradas, para permitir a comparação entre os diferentes trabalhos de forma mais confiável.

Em quase a totalidade dos trabalhos avaliados, o material vegetal acondicionado nas bolsas de decomposição foram apenas compostos por folhas, e apenas um trabalho dentre os 13 avaliados utilizou outras partes vegetais componentes da serapilheira, onde Laclau et al. (2010) estudaram a decomposição de resíduos da colheita em área de plantio de eucalipto, em que constataram que a decomposição das folhas e raízes finas foi mais rápida do que a decomposição galhos e raízes mais grossas.

Além disso, plantas do bioma Cerrado possuem um crescimento subterrâneo bastante significativo, e em alguns locais, a maior parte da biomassa se encontra alocada no subsolo 
(LOIOLA; SCHERER-LORENZEN; BATALHA, 2015). Tendo em vista tal fato, cabe ressaltar que nenhum dos trabalhos avaliou a quantificação da decomposição das raízes de espécies nativas do Cerrado, o que poderia gerar dados para o melhor entendimento da dinâmica da ciclagem de nutrientes nestes ecossistemas.

Sendo assim, uma vez que as folhas representam, em média, $70 \%$ ou mais da constituição da serapilheira, a velocidade e as taxas de decomposição apresentadas na grande maioria dos artigos podem estar sendo superestimadas, pois as outras partes componentes da serapilheira não estão sendo avaliadas, e estas por sua vez, possuem composições químicas e velocidades de decomposição bastante distintas (LACLAU et al., 2010).

Tabela 5 - Quantificação dos trabalhos de acordo com a massa de material vegetal acondicionada nas bolsas de decomposição.

Table 5 - Quantification of the works according to the mass of vegetal material placed in the decomposition bags.

\begin{tabular}{lcc}
$\begin{array}{c}\text { Massa } \\
\text { acondiconada nas } \\
\text { litter bags }\end{array}$ & $\mathbf{N}$ & Referências \\
\hline $\mathbf{5}$ g & 5 & $\begin{array}{c}\text { Carvalho et al. (2014); Freitas; Cianciaruso; Batalha (2012); Bambi et al. 2011; } \\
\text { Valenti; Cianciaruso; Batalha (2008); Cianciaruso et al. (2006) }\end{array}$ \\
\hline $\mathbf{1 0}$ g & 4 & $\begin{array}{c}\text { Villalobos-Veja et al. (2011); Kozovits et al. (2007); Silva; Poggiani; Laclau (2011); } \\
\text { Laclau et al. (2010) }\end{array}$ \\
\hline $\mathbf{2 0}$ g & 1 & Oliveira et al. (2017) \\
\hline $\mathbf{2}$ a $\mathbf{5}$ g & 1 & Sanches et al. (2009) \\
\hline $\mathbf{3}$ a $\mathbf{5}$ g & 1 & Silva et al. (2009) \\
\hline $\mathbf{2}$ (tea bag) & 1 & Miatto e Batalha (2016);
\end{tabular}

Em que: $\mathrm{N}$ = Número de trabalhos.

Dentre as diferentes frações da serapilheira, os galhos e ramos são responsáveis por aproximadamente $20 \%$ do total de materiais depositados sobre os solos florestais, e estes possuem altos teores de lignina. Pegoraro et al. (2011) afirmam que a lignina é um importante componente da parede celular das árvores, sendo este um biopolímero abundante e que fornece carbono ao solo de forma expressiva, pois apenas alguns microrganismos são capazes de decompô-la devido a sua alta complexidade estrutural, contribuindo assim, substancialmente, para a formação de carbono estável no solo.

Os tempos de coleta das bolsas a campo apresentaram também discrepâncias metodológicas entre os trabalhos, sendo que a data da primeira coleta variou entre duas semanas de exposição das bolsas a campo como nos trabalhos de Bambi et al. (2011) e Silva et al. (2009) com entre 14 e 15 dias, respectivamente, indo até o extremo de cinco meses de exposição para a primeira e única coleta como observado no trabalho dos autores Kozovits et al. (2007). A grande maioria dos trabalhos fez a primeira coleta com 30 dias de exposição das bolsas à decomposição, como nos trabalhos: Oliveira et al. (2017); Carvalho et al. (2014); Freitas, Cianciaruso e Batalha, (2012); Silva, Poggiani e Laclau, (2011); Laclau et al. (2010); Valenti, Cianciaruso e Batalha (2008); Cianciaruso et al. (2006).

De forma geral, a decomposição da serapilheira tem comportamento exponencial de decaimento, em que os primeiros dias de exposição apresentam as maiores taxas de decomposição, devido ao fato da rápida degradação dos elementos mais facilmente decomponíveis (celulose e holocelulose), restando apenas os elementos com estruturas e composições químicas mais complexas, como é o caso da fração húmica que possui decomposição mais lenta (GOYA et al., 2008). 
Tendo em vista tais padrões de decomposição, os trabalhos que apresentaram a primeira coleta de forma tardia podem ter estimado suas curvas de decomposição de forma não representativa à realidade, não sendo provavelmente percebidos com clareza os pontos de inflexão da curva de decomposição. Esta diferença entre os tempos de coleta evidenciada na análise dos trabalhos, novamente ratifica a necessidade de uma padronização das metodologias, para que a comparação entre os trabalhos possa ser realizada de forma mais segura.

Com relação ao tempo total de análise e exposição das bolsas à decomposição, os seus resultados podem ser visualizados na Tabela 6, na qual oito dos 13 trabalhos tiveram duração máxima de um ano, com apenas dois trabalhos publicados em que o tempo total de análise foi superior a um ano, e apenas um trabalho com mais de um ano e meio, como foi observado no trabalho de Laclau et al. (2010), com experimento realizado em área de plantios de eucalipto no Brasil e no Kongo. Outros dois trabalhos apresentaram tempo total de experimento inferior a um ano, com foi o caso de Kozovits et al. (2007) e Carvalho et al. (2014) com cinco e seis meses, respectivamente.

Publicações contendo estudos de longa duração para a decomposição da serapilheira no bioma Cerrado não foram encontrados, sendo assim este um ponto a ser explorado nas futuras pesquisas, verificando a existência ou não de possíveis alterações interanuais entre as taxas de decomposição, uma vez que diversos fatores afetam a degradação dos materiais, tais como: umidade, temperatura, constituição da serapilheira e atividade e diversidade biológica do solo (SANTANA et al., 2011; SOUTO et al., 2009).

Tabela 6 - Classificação dos manuscritos avaliados quanto ao tempo total de exposição das bolsas de decomposição.

Table 6 - Classification of the evaluated manuscripts regarding the total time of exposure of the decomposition bags.

\begin{tabular}{lcc}
\hline Tempo total do experimento & $\mathbf{N}$ & Referências \\
\hline $\mathbf{3}$ meses (tea bag) & 1 & Miatto e Batalha (2016) \\
\hline $\mathbf{5}$ meses & 1 & Kozovits et al. (2007) \\
\hline $\mathbf{1}$ ano & 8 & $\begin{array}{c}\text { Oliveira et al. (2017); Freitas; Cianciaruso; Batalha (2012); Bambi et al. } \\
(2011) ; \text { Silva; Poggiani; Laclau (2011); Sanches et al. (2009); Silva et al. } \\
\text { (2009); Cianciaruso et al. (2006) }\end{array}$ \\
\hline $\mathbf{1}$ ano e $\mathbf{2}$ meses & 1 & Villalobos-Vega et al. (2011) \\
\hline $\mathbf{1 , 5}$ e 2 anos & 1 & Laclau et al. (2010) \\
\hline
\end{tabular}

Em que: $\mathrm{N}=$ Número de trabalhos.

Entre as várias formas fisionômicas de vegetação nativa ocorrentes no Cerrado, fatores determinantes para estas diferenciações são: profundidade efetiva do solo, presença de concreções no perfil, proximidade à superfície do lençol freático, drenagem e fertilidade. Além das variações na fisionomia, ocorrem alterações também na composição florística, fitossociologia e produtivi dade desses ecossistemas, em decorrência das variações nas características químicas e físicas dos solos (HARIDASAN, 2000).

Sendo assim, trabalhos sobre a quantificação da produção e decomposição da serapilheira nas demais fitofisionomias que ainda não possuem estudos publicados se tornam essenciais para o melhor entendimento da dinâmica destes ecossistemas. Vale ressaltar também que estudos comparando diferentes metodologias aplicadas nos distintos ecossistemas do bioma Cerrado, com o intuito de verificar quais os procedimentos mais adequados a cada situação, os quais possibilitem 
também a comparação entre os trabalhos dentre uma mesma formação fisionômica e entre diferentes fitofisionomias são também indicados.

\section{Considerações finais}

Os trabalhos avaliados referentes à temática de decomposição da serapilheira, com exceção a um trabalho desenvolvido sobre os resíduos da colheita de plantios de eucalipto, não apresentaram avaliações da decomposição de outros materiais vegetais componentes da serapilheira, que não as folhas, isto pode implicar em uma superestimação da velocidade de decomposição destes, sendo assim, é necessária a realização de estudos que visem a este tipo de investigação, em especial nas áreas de Cerrado nativo.

Dentre as diferentes fitofisionomias do Cerrado, pôde-se observar que algumas delas, como: veredas, palmeirais, parque de Cerrado, campo sujo, campo rupestre e campo limpo, ainda não possuem nenhum tipo de trabalho referente à produção e decomposição da serapilheira, e ainda, a escassez de trabalhos com monitoramentos de longo prazo desta temática apresentam-se como uma linha de pesquisa a ser seguida, uma vez que estudos com breve duração não permitem avaliar os efeitos de alterações sazonais e interanuais que possivelmente estejam correlacionadas com as mudanças climáticas.

A falta de estudos comparativos entre as metodologias apresenta um caminho para pesquisas futuras, pois o preenchimento desta lacuna implicará na possibilidade de comparação dos trabalhos realizados em ambientes semelhantes de forma mais confiável, e até mesmo entre ecossistemas diferentes, caso suas metodologias permitam tais análises.

De forma geral, as metodologias utilizadas nos trabalhos de aporte e decomposição da serapilheira apresentaram alto grau de variação entre si, evidenciando assim, a necessidade da criação de um protocolo único, o qual permitiria a comparação dos estudos, sendo que este deve ser construído com base nos trabalhos já realizados e com encontros dos pesquisadores da área para debater quais os melhores procedimentos.

Com base nos artigos avaliados, sugere-se como metodologia para avaliação da produção de serapilheira que o formato de coletores deva ser quadrado, pois facilita a extrapolação para um hectare, com as frações da serapilheira definidas em: folhas, galhos, partes reprodutivas e miscelânea e com um número mínimo de 10 coletores. Já para os estudos de decomposição, sugere-se litter bags com dimensões de $20 \times 20 \mathrm{~cm}$ e malha com abertura de $2 \mathrm{~mm}$, com acondicionamento de diferentes matérias (não somente folhas) para se ter uma estimativa mais real das taxas de decomposição da serapilheira como um todo, além de estudos com mais de um ano de avaliação, para evidenciar os efeitos das possíveis alterações interanuais.

\section{Referências}

ALMEIDA, J. C. R. et al. A positive growth response to $\mathrm{NaCl}$ applications in Eucalyptus plantations established on K-deficient soils. Forest Ecology and Management, Amsterdam, v. 259 , p. 1786-1795, 2010.

AQUINO, P. S. R. et al. Análise espacial da produtividade de serapilheira em uma mata de galeria. Ciência Florestal, Santa Maria, v. 26, n. 2, p. 489-500, 2016.

BALCH, J. et al. Negative fire feedback in a transitional forest of southeastern Amazonia. Global Change Biology, Nova Jersey, v. 14, p. 2276-2287, 2008.

BAMBI, P. et al. Decomposição e redistribuição de nutrientes das folhas de espécies da floresta de transição Amazônia - Cerrado, MT. Ciência e Natura, Santa Maria, v. 33, n. 1, p. 17-31, 2011.

BASTOS, L. A.; FERREIRA, I. M. Composições fitofisionômicas do bioma cerrado: estudo sobre o subsistema de Vereda. Espaço em Revista, Catalão, v. 12, n. 2, p. 97-108, 2010.

BORENSTEIN, M. et al. Introduction to metaanalysis. Londres: Wiley, 2009. 421 p. 
BUSTAMANTE, M. M. C. et al. Potential impacts of climate change on biogeochemical functioning of Cerrado ecosystems. Brazilian Journal of Biology, São Carlos, v. 72, n. 3, p. 655$671,2012$.

BUTLER, A. et al. Annual variation in soil respiration and its component parts in two structurally contrasting woody savanas in Central Brazil. Plant and Soil, Heidelberg, v. 352, p. 129-142, 2012.

CARVALHO, G. H. et al. Are fire, soil fertility and toxicity, water availability, plant functional diversity, and litter decomposition related in a Neotropical savanna? Oecologia, Heidelberg, v. 175, p. 923-935, 2014.

CIANCIARUSO, M. V. et al. Produção de serapilheira e decomposição do material foliar em um cerradão na Estação Ecológica de Jataí, município de Luiz Antônio, SP, Brasil. Acta Botanica Brasilica, Feira de Santana, v. 20, n. 1, p. 49-59, 2006.

COSTA, C. C. A. et al. Análise comparativa da produção de serrapilheira em fragmentos arbóreos e arbustivos em área de caatinga na Flona de Açu - RN. Revista Árvore, Viçosa, MG, n. 34, v. 2, p. 259-265, 2010.

EPRON, D. et al. Do changes in carbon allocation account for the growth response to potassium and sodium applications in tropical. Tree Physiology, Oxford, v. 32, p. 667-679, 2011.

EPRON, D. et al. Partitioning of net primary production in Eucalyptus and Acacia stands and in mixed-species plantations: Two case-studies in contrasting tropical environments. Forest Ecology and Management, Amsterdam, v. 301, p. 102-111, 2012.

FONTES, M. P. F. Intemperismo de rochas e minerais. In: KER, J. C.; CURI, N.; SCHAEFER, C. E. G. R.; VIDAL-TORRADO, P. (ed.). Pedologia: fundamentos. Viçosa, MG: SBCS, 2012, p. 171202.

FREITAS, J. R.; CIANCIARUSO, M. V.; BATALHA, M. A. Functional diversity, soil features and community functioning: a test in a cerrado site. Brazilian Journal of Biology, São Carlos, v. 72, n. 3, p. 463-470, 2012.

GIÁCOMO, R. G.; PEREIRA, M. G.; MACHADO, D. L. Aporte e decomposição de serapilheira em áreas de cerradão e mata mesofítica na estação ecológica de pirapitinga - MG. Ciência Florestal, Santa Maria, v. 22, n. 4, p. 669-680, 2012.

GOYA, J. F. et al. Decomposition and nutrient release from leaf litter in Eucalyptus grandis plantations on three different soils in Entre Ríos, Argentina. Revista Bosque, Valdivia, v. 29, n. 3, p. 217-226, 2008.

HARIDASAN, M. Nutrição mineral de plantas nativas do cerrado. Revista Brasileira de Fisiologia Vegetal, Londrina, v. 12, n. 1, p. 54-64, 2000.

KEUSKAMP J. A. et al. Tea Bag Index: a novel approach to collect uniform decomposition data across ecosystems. Methods. Ecology and Evololution, London, v. 4, p. 1070-1075, 2013.

KOZOVITS, A. R. et al. Nutrient resorption and patterns of litter production and decomposition in a Neotropical Savanna. Functional Ecology, Londres, v. 21, p. 1034-1043, 2007.

LACLAU, J. P. et al. Biogeochemical cycles of nutrients in tropical Eucalyptus plantations Main features shown by intensive monitoring in Congo and Brazil. Forest Ecology and Management, Amsterdam, v. 259, p. 1771-1785, 2010.

LATHWELL, D. J.; GROVE, T. L. Soil-Plant Relationships in the Tropics. Annual Review of Ecology and Systematics, Palo alto, v. 17, p. 1-16, 1986.

LOIOLA, P. P.; SCHERER-LORENZEN, M.; BATALHA, M. A. The role of environmental filters and Functional traits in predicting the root biomass and productivity in savannas and tropical seasonal forests. Forest Ecology and Management, Amsterdam, v. 342, p. 49-55, 2015. 
LOPES, J. F. B. et al. Deposição e decomposição de serapilheira em área da Caatinga. Revista Agro@mbiente On-line, Boa Vista, v. 3, n. 2, p. 72-79, 2009.

MIATTO, R. C.; BATALHA, M. A. Leaf chemistry of woody species in the Brazilian cerrado and seasonal forest: response to soil and taxonomy and effects on decomposition rates. Plant Ecology, Oxford, v. 217, p. 1467-1479, 2016.

NASCIMENTO, L. S., CERQUEIRA, R. M., HENDERSON, B. L. R. Produção de serapilheira em um fragmento adjacente a uma cava de mineração, Ribeirão Grande, SP. Revista Brasileira de Engenharia Agrícola e Ambiental, Campina Grande, v. 19, n. 9, p. 892-897, 2015.

OLIVEIRA, B. et al. Unraveling the ecosystem functions in the Amazonia-Cerrado transition: evidence of hyperdynamic nutrient cycling. Plant Ecology, Oxford, n. 218, p. 225-239, 2017.

PAIVA, A. O.; SILVA, L. C. R.; HARIDASAN, M. Productivity-efficiency tradeoffs in tropical gallery forestsavanna transitions: linking plant and soil processes through litter input and composition. Plant Ecology, Oxford, v. 216, p. 775-787, 2015.

PARRON, L. M.; BUSTAMANTE, M. M. C.; MARKEWITZ, D. Fluxes of nitrogen and phosphorus in a gallery forest in the Cerrado of central Brazil. Biogeochemistry, Heidelberg, v. 105, n. 1-3, p. 89-104, 2011.

PEGORARO, R. F.etal. Fenóis derivados da lignina, carboidratos e aminoaçúcares em serapilheira e solos cultivados com eucalipto e pastagem. Revista Árvore, Viçosa, MG, v. 35, n. 2, p. 359-370, 2011.

PICKERING, C. M.; BYRNE, J. The benefits of publishing systematic quantitative literature reviews for PhD candidates and other early career researchers. Higher Education Research and Development, Londres, v. 33, p. 534-548, 2014.

POGGIANI, F. Estoques e transferências de nutrientes nos ecossistemas. In: MARTINS, S. V. Ecologia de florestas tropicais do Brasil. Viçosa, MG: Editora UFV, 2012. p. 175-242.

QUESADA, C. A. et al. Seasonal variations in soil water in two woodland savannas of central Brazil with different fire history. Tree Physiology, Oxford, v. 28, p. 405-415, 2008.

RIBEIRO, J. F.; WALTER, B. M. T. As principais fitofisionomias do Bioma Cerrado. In.: SANO, S. M.; ALMEIDA, S. P.; RIBEIRO, J. F. Ecologia e flora. Brasília: EMBRAPA, 2008. p. 152-212.

RUBIM, P.; NASCIMENTO, H. E. M.; MORELLATO, L. P. C. Variações interanuais na fenologia de uma comunidade arbórea de floresta semidecídua no sudeste do Brasil. Acta Botânica Brasilica, São Paulo, v. 24, n. 3, p. 756-762, 2010.

SANCHES, L. et al. Dinâmica sazonal da produção e decomposição de serrapilheira em floresta tropical de transição. Revista Brasileira de Engenharia Agrícola e Ambiental, Campina Grande, v. 13, n. 2, p. 183-189, 2009.

SANTANA, J. A. S. et al. Decomposição da biomassa foliar de cana-de açúcar em um neossolo na região de areia - PB. Revista Caatinga, Mossoró, v. 24, n. 3, p. 28-32, 2011.

SCORIZA, R. N. et al. Métodos para coleta e análise de serapilheira aplicados à ciclagem de nutrientes. Floresta e Ambiente, Seropédica, v. 2, n. 2, p. 01-18, 2012.

SETALA, H.; MARSHALL, V. G.; TROFYMOW, J. A. Influence of body size of soil fauna on litter decomposition and $15 \mathrm{~N}$ uptake by poplar in a pot trial. Soil Biology and Biochemistry, Amsterdam, v. 28, n. 12, p. 1661-1675, 1996.

SILVA, C. J. et al. Contribuição de folhas na formação da serrapilheira e no retorno de nutrientes em floresta de transição no norte de Mato Grosso. Acta amazônica, Manaus, v. 39, n. 3, p. 591$600,2009$. 
SILVA, C. J. et al. Produção de serrapilheira no Cerrado e Floresta de Transição AmazôniaCerrado do Centro-Oeste Brasileiro. Acta amazônica, Manaus, v. 37, n.4, p. 543-548, 2007.

SILVA, P. H. M.; POGGIANI, F.; LACLAU, J. P. Applying sewage sludge to eucalyptus grandis plantations: effects on biomass production and nutrient cycling through litterfall. Applied and Environmental Soil Science, London, v. 2011, p. 1-11, 2011.

SOUTO, P. C. et al. Características químicas da serapilheira depositada em área de caatinga. Revista Caatinga, Mossoró, v. 22, n. 1, p. 264-272, 2009.

VALENTI, M. W.; CIANCIARUSO, M. V.; BATALHA, M. A. Seasonality of litterfall and leaf decomposition in a cerrado site. Brazilian Journal of Biology, São Carlos, v. 68, n. 3, p. 459-465, 2008.

VILLALOBOS-VEJA, R. et al. Leaf litter manipulations alter soil physicochemical properties and tree growth in a Neotropical savana. Plant and Soil, Heidelberg, v. 346, p. 385-397, 2011.

ZIMMERMAN, J. K. et al. Flowering and fruiting phenologies of seasonal and aseasonal neotropical forests: the role of annual changes in irradiance. Journal of Tropical Ecology, Cambridge, v. 23, p. 231-251, 2007.

WIEDER, R. K.; LANG, G. E. A critique of the analytical methods used examining decomposition data obtained from litter bags. Ecology, Washington, v. 63, p. 1636-1642, 1982.

WILCKE, W.; LILIENFEIN, J. Biogeochemical consequences of the transformation of native Cerrado into Pinus caribaea plantations in Brazil. Plant and Soil, Heidelberg, v. 238, p. 175-189, 2002. 